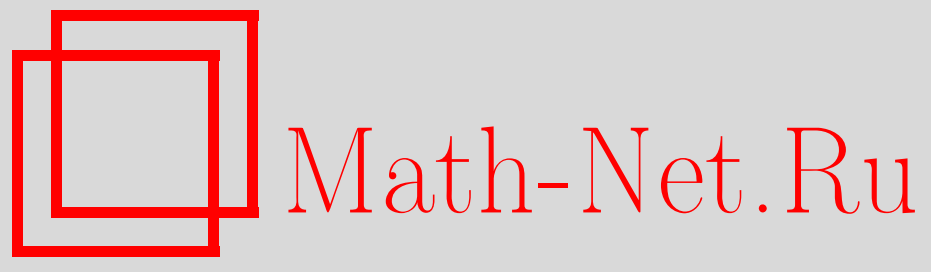

А. С. Гордиенко, Коразмерности функциональных тождеств, УМH, 2009, том 64, выпуск 1, 141-142

DOI: https://doi.org/10.4213/rm9267

Использование Общероссийского математического портала Math-Net.Ru подразумевает, что вы прочитали и согласны с пользовательским соглашением http://www . mathnet.ru/rus/agreement

Параметры загрузки:

IP: 54.210 .77 .194

26 апреля 2023 г., 16:42:10

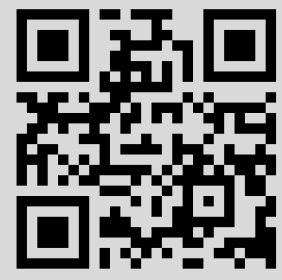




\section{Коразмерности функциональных тождеств}

\section{А. С. Гордиенко}

В середине 1980-х годов были сформулированы гипотезы С. А. Амицура и А. Регева об асимптотическом поведении коразмерностей тождеств. В случае обычных тождеств гипотеза Амицура была доказана в 1999 г. М. В. Зайцевым и А. Джамбруно [1] для всех ассоциативных алгебр над полями характеристики 0. В 2008 г. вышла работа А. Регева и А. Берела [2], в которой для обычных коразмерностей широкого класса алгебр доказывается гипотеза Регева. Наряду с обычными тождествами, в теории колец находят применение и функциональные тождества [3]. В связи с этим возникает вопрос о справедливости указанных гипотез для функциональных коразмерностей.

Пусть $A$ - ассоциативная алгебра над полем $F$ произвольной характеристики. Выражение $f=\sum_{i=1}^{n}\left(G_{i}^{i}\left(\bar{x}_{n}\right) x_{i} a_{i}+b_{i} x_{i} H_{i}^{i}\left(\bar{x}_{n}\right)\right)$ называется полилинейным функционалъным многочленом степени $n$ с коэффициентами в алгебре $A$. Здесь $G_{i}, H_{i}$ : $A^{\otimes(n-1)} \rightarrow A$ - произвольные $F$-линейные отображения, для таких отображений $Q$ мы используем сокращение $Q^{i}\left(\bar{x}_{n}\right)=Q\left(x_{1}, \ldots, x_{i-1}, x_{i+1}, \ldots, x_{n}\right) ; a_{i}, b_{i} \in A \cup\{1\}$; $1 \leqslant i \leqslant n ; n \geqslant 2$. Если $f\left(p_{1}, \ldots, p_{n}\right)=0$ для всех $p_{1}, \ldots, p_{n} \in A$, то говорят, что $f-$ функииональное тождество алгебры $A$. Понятно, что множество $\operatorname{FId}_{n}(A)$ полилинейных функциональных тождеств степени $n$ является линейным подпространством в пространстве $F P_{n}(A)$ всех полилинейных функциональных многочленов степени $n$. Коразмерности $f c_{n}(A)=\operatorname{dim} \frac{F P_{n}(A)}{\operatorname{FId}_{n}(A)}$ функциональных тождеств называются функииональными коразмерностями алгебры $A$.

Teоpema 1. Пусть $A^{2}=\langle a b \mid a, b \in A\rangle_{F}$. Eсли $A^{2}=0$, mo $f c_{n}(A)=0$. Ecлu $A^{2} \neq 0 u \operatorname{dim} A=\infty$, mo $f c_{n}(A)=\infty$ для всех $n \geqslant 2$. Eсли $A^{2} \neq 0 u \operatorname{dim} A<\infty$, mo $(\operatorname{dim} A)^{n-1} \leqslant f c_{n}(A) \leqslant \operatorname{dim}\left(A^{2}\right) \cdot(\operatorname{dim} A)^{n}$.

Следствие. Если $\operatorname{dim} A<\infty$, то существует $\lim _{n \rightarrow \infty} \sqrt[n]{f c_{n}(A)}=\operatorname{dim} A \in \mathbb{Z}_{+}$, m.e. для $f c_{n}(A)$ выполняется гипотеза С. А. Амииура.

Tеорема 2. Если $\operatorname{dim} A<\infty$ и существует такой элемент $a \in A$, что $A^{2}=$ $A a+a A+A a A, \operatorname{mo} f c_{n}(A) \sim \operatorname{dim}\left(A^{2}\right) \cdot(\operatorname{dim} A)^{n} n p u n \rightarrow \infty$.

СледствиЕ. Если алгебра $A$ содержит единицу, то $f c_{n}(A) \sim(\operatorname{dim} A)^{n+1} n p u$ $n \rightarrow \infty$. Таким образом, для $f c_{n}(A)$ справедлива гипотеза А. Регева.

ДоказАТеЛЬСтво теоремы 1 . Если $A^{2}=0$, то $F P_{n}(A)=\operatorname{FId}_{n}(A)$ и $f c_{n}(A)=0$. Поэтому считаем, что $A^{2} \neq 0$. Тогда существуют такие $a, b \in A$, что $a b \neq 0$. Пусть для некоторого $m \in \mathbb{N}$ существуют линейно независимые элементы $e_{j} \in A, 1 \leqslant j \leqslant m$. Обозначим через $\Lambda$ множество наборов $k=\left(k_{1}, \ldots, k_{n-1}\right)$, в которых $1 \leqslant k_{\ell} \leqslant m$ при $1 \leqslant \ell \leqslant n-1$. Для каждого $k \in \Lambda$ определим полилинейное отображение $G_{k}$ так, что

$$
G_{k}\left(e_{i_{1}}, \ldots, e_{i_{n-1}}\right)= \begin{cases}a, & \text { если } i_{\ell}=k_{\ell} \text { для всех } \ell \\ 0 & \text { в противном случае. }\end{cases}
$$

Тогда функциональные многочлены $f_{k}=G_{k}^{n}\left(\bar{x}_{n}\right) x_{n}, k \in \Lambda$, линейно независимы по модулю тождеств. Действительно, если $\sum_{k \in \Lambda} \alpha_{k} f_{k} \equiv 0$ для некоторых $\alpha_{k} \in F$, то, зафиксировав $t=\left(t_{1}, \ldots, t_{n-1}\right) \in \Lambda$ и подставив $x_{1}=e_{t_{1}}, \ldots, x_{n-1}=e_{t_{n-1}}, x_{n}=b$,

Работа поддержана грантами РФФИ № 06-01-00485 и НШ-1983.2008.1. 
мы получим, что все $f_{k}$, за исключением $f_{t}$, обратятся в 0 . Отсюда $\alpha_{t}=0$. Таким образом, $f c_{n}(A) \geqslant|\Lambda|=m^{n-1}$. Поэтому если $\operatorname{dim} A<\infty$, то $f c_{n}(A) \geqslant(\operatorname{dim} A)^{n-1}$. Если же $\operatorname{dim} A=\infty$, то $f c_{n}(A)=\infty$.

Пусть $\operatorname{dim} A<\infty$. Заметим, что всякий функциональный многочлен можно рассматривать как полилинейное отображение от $n$ аргументов, принимающее значения в пространстве $A^{2}$. Таким образом, определено естественное линейное отображение $\pi: F P_{n}(A) \rightarrow \operatorname{Hom}_{\mathrm{F}}\left(A^{\otimes n} ; A^{2}\right)$, где $\operatorname{Hom}_{\mathrm{F}}\left(A^{\otimes n} ; A^{2}\right)$ - пространство всех полилинейных отображений из $A$ в $A^{2}$ от $n$ аргументов. Понятно, что $\operatorname{ker} \pi=\operatorname{FId}_{n}(A)$. Отсюда следует, что существует вложение $\frac{F P_{n}(A)}{\operatorname{FId}_{n}(A)} \subseteq \operatorname{Hom}_{\mathrm{F}}\left(A^{\otimes n} ; A^{2}\right)$. Поэтому

$$
f c_{n}(A) \leqslant \operatorname{dim} \operatorname{Hom}_{\mathrm{F}}\left(A^{\otimes n} ; A^{2}\right)=\operatorname{dim}\left(A^{2}\right) \cdot(\operatorname{dim} A)^{n} .
$$

ДокАЗАТЕльство теоремы 2. Дополним $a$ до некоторого базиса $e_{1}, \ldots, e_{m-1}$, $a=e_{m}$ алгебры $A$. Пусть $\varphi_{1}, \ldots, \varphi_{m}$ - двойственный базис в $A^{*}$, т. е. $\varphi_{i}\left(e_{j}\right)=\delta_{i j}$. По условию в $A^{2}$ можно выбрать базис $w_{1}, \ldots, w_{q}$, состоящий из элементов вида $e_{k} a e_{\ell}$, $e_{k} a, a e_{\ell}$. Определим отображения $H_{t}(x)$ вида $e_{k} x e_{\ell}, e_{k} x, x e_{\ell}$ так, чтобы $H_{t}(a)=w_{t}$ для всех $1 \leqslant t \leqslant q$. В силу равенства $\operatorname{Hom}_{\mathrm{F}}\left(A^{\otimes n} ; A^{2}\right)=\left(A^{*}\right)^{\otimes n} \otimes A^{2}$ отображения $\varphi_{j_{1}}\left(x_{1}\right) \cdots \varphi_{j_{n}}\left(x_{n}\right) w_{t}$, где $j_{1}, \ldots, j_{n} \in\{1, \ldots, m\}, 1 \leqslant t \leqslant q$, образуют базис в $\operatorname{Hom}_{\mathrm{F}}\left(A^{\otimes n} ; A^{2}\right)$. Введем на наборах $\left(j_{1}, \ldots, j_{n}, t\right)$ лексикографическое упорядочение. Тогда, например, $H_{t}(x)$ как элемент пространства $\operatorname{Hom}_{\mathrm{F}}\left(A ; A^{2}\right)$ будет суммой $\varphi_{m}(x) w_{t}$ и младших членов $\varphi_{i}(x) w_{j}, i<m$. Пусть $M_{1} \subseteq \operatorname{Hom}_{\mathrm{F}}\left(A ; A^{2}\right)$ - линейная оболочка отображений вида $e_{k} x e_{\ell}, e_{k} x, x e_{\ell}$. Тогда подпространство $M=\frac{F P_{n}(A)}{\operatorname{Fdd}_{n}(A)}$ является линейной оболочкой отображений $\varphi_{j_{1}}\left(x_{1}\right) \cdots \varphi_{j_{i-1}}\left(x_{i-1}\right) \varphi_{j_{i+1}}\left(x_{i+1}\right) \cdots \varphi_{j_{n}}\left(x_{n}\right) H\left(x_{i}\right)$, где $1 \leqslant i \leqslant n$, а $H \in M_{1}$. Рассмотрим факторпространство $\operatorname{Hom}_{\mathrm{F}}\left(A^{\otimes n} ; A^{2}\right) / M$. Если в наборе $\left(j_{1}, \ldots, j_{n}, t\right)$ для некоторого $i$ выполняется равенство $j_{i}=m$, то соответствующий элемент $f=\varphi_{j_{1}}\left(x_{1}\right) \cdots \varphi_{j_{n}}\left(x_{n}\right) w_{t}$ можно проредуцировать по модулю $M$ к линейной комбинации базисных элементов пространства $\operatorname{Hom}_{F}\left(A^{\otimes n} ; A^{2}\right)$, которым соответствуют меньшие наборы. Для этого достаточно вычесть $g=\varphi_{j_{1}}\left(x_{1}\right) \cdots \varphi_{j_{i-1}}\left(x_{i-1}\right) \times$ $\varphi_{j_{i+1}}\left(x_{i+1}\right) \cdots \varphi_{j_{n}}\left(x_{n}\right) H_{t}\left(x_{i}\right) \in M$, так как $f$ будет для $g$ в выбранном упорядочении старшим членом. Производя редукции, мы получим, что $\operatorname{Hom}_{\mathrm{F}}\left(A^{\otimes n} ; A^{2}\right) / M$ является линейной оболочкой образов элементов, которым соответствуют наборы $\left(j_{1}, \ldots, j_{n}, t\right)$, где $j_{i} \neq m$ для всех $i$. Следовательно, $\operatorname{dim} \operatorname{Hom}_{\mathrm{F}}\left(A^{\otimes n} ; A^{2}\right) / M \leqslant \operatorname{dim}\left(A^{2}\right) \cdot(\operatorname{dim} A-1)^{n}$ и

$$
\begin{aligned}
f c_{n}(A) & =\operatorname{dim} M=\operatorname{dim} \operatorname{Hom}_{\mathrm{F}}\left(A^{\otimes n} ; A^{2}\right)-\operatorname{dim} \operatorname{Hom}_{\mathrm{F}}\left(A^{\otimes n} ; A^{2}\right) / M \\
& \geqslant \operatorname{dim}\left(A^{2}\right) \cdot(\operatorname{dim} A)^{n}-\operatorname{dim}\left(A^{2}\right) \cdot(\operatorname{dim} A-1)^{n} .
\end{aligned}
$$

Используя верхнюю оценку из теоремы 1 и устремляя $n$ к $\infty$, получаем требуемую асимптотику.

\section{Список литературы}

[1] M. V. Zaicev, A. Giambruno, Polynomial identities and asymptotic methods, Math. Surveys Monogr., 122, Amer. Math. Soc., Providence, RI, 2005. [2] A. Berele, A. Regev, Trans. Amer. Math. Soc., 360:10 (2008), 5155-5172. [3] К. И. Бейдар, А. В. Михалёв, М. А. Чеботарь, УМН, 59:3 (2004), 3-30; англ. пер.: K. I. Beidar, A. V. Mikhalev, M. A. Chebotar, Russian Math. Surveys, 59:3 (2004), 403-428.

А. С. Гордиенко (А.S. Gordienko)

Московский государственный университет им. М. В. Ломоносова

E-mail: gordienko_a_s@mail.ru
Представлено А. В. Михалёвым Принято редколлегией 07.12 .2008 